\title{
Idealizando Jogos Digitais de Pensamento Computacional a Partir do Bebras Challenge: Um Estudo Exploratório.
}

\author{
Jéssica Laisa ${ }^{1}$, Eduardo Henrique ${ }^{1}$, Wendell Oliveira ${ }^{1}$ \\ ${ }^{1}$ Programa de Pós-Graduação em Sistemas Computacionais - PPgSC \\ Universidade Federal do Rio Grande do Norte - UFRN \\ Campus Universitário Lagoa Nova -Natal/RN - Brasil \\ jessicalaisajl@gmail.com, eduardoaranha@dimap.ufrn.br, \\ wendell.cmd@gmail.com
}

\begin{abstract}
National and international initiatives have been incorporating computational thinking in basic education. One of them is the Bebras Challenge, which presents a series of questions that exercise and evaluate computational thinking. In the context of this initiative, we present an exploratory study carried out with 19 students graduating from a federal university in order to investigate whether the Bebras issues are good sources for game design construction. With this, this work intends to answer the following research questions: What is the quality of game designs produced? What is the effort required to produce game designs? What are the difficulties presented by the students? The results show that it is possible to develop good game designs based on Bebras issues, which are a good source of inspiration.
\end{abstract}

Resumo. Iniciativas nacionais e internacionais vêm inserindo pensamento computacional no ensino básico. Uma delas é o Bebras Challenge, que apresenta uma série de questões, que exercitam e avaliam o pensamento computacional. No contexto dessa iniciativa, apresentamos um estudo exploratório realizado com 19 alunos graduandos de uma universidade federal, com o objetivo de investigar se as questões do Bebras são boas fontes para construção de game design. Com isso, este trabalho pretender responder as seguintes questões de pesquisa: Qual a qualidade dos game designs produzidos? Qual o esforço necessário para realizar a produção dos game designs? Quais as dificuldades apresentadas pelos alunos? Os resultados alcançados mostram que é possível desenvolver bons game designs a partir das questões do Bebras, mostrando-se estas uma boa fonte de inspiração.

\section{Introdução}

Cada vez mais, a tecnologia adentra nas instituições de ensino e é uma forte ferramenta para estimular o desenvolvimento dos alunos, propiciando engajamento, motivação e auxiliando os professores nos trabalhos em sala de aula. O autor Kenski (2013) ressalta que assim como a educação, a tecnologia também é poder. E dentre as tecnologias usadas nas escolas uma que tem grande potencial de engajamento e participação dos alunos estão os jogos digitais. [Prensky, 2001] relata que a utilização de jogos digitais na educação traz grandes vantagens, como a possibilidade de motivar o jogador, pois um aluno se divertindo aprende melhor. 
Porém, antes dos jogos educativos digitais serem desenvolvidos, eles passam por um processo de criação e uma delas é logo no inicio, o desenvolvimento do documento de game design. [Leite e Mendonça, 2013] afirmam a importância da definição do documento de game design, o qual contém as especificações do jogo e onde são descritas e explicadas às características principais do jogo, como jogabilidade, controles, interfaces, personagens, armas, golpes, inimigos, fases e demais aspectos gerais do projeto.

O objetivo deste artigo é trazer um estudo exploratório que verificar como atividades ou testes de habilidades podem ser boas fontes para construção de game design para se trabalhar pensamento computacional. Nesse contexto, este trabalho levanta as seguintes questões: Qual a qualidade dos game designs produzidos? Qual o esforço necessário para realizar a produção dos game designs? Quais as dificuldades apresentadas pelos alunos?

Para atingir esse objetivo, realizamos neste artigo um estudo exploratório com alunos de graduação que idealizaram jogos digitais educativos a partir de questões do teste internacional BEBRAS, que trabalha com pensamento computacional.

Sendo assim, as próximas seções deste trabalho estão organizadas da seguinte maneira: a Seção 2 discute sobre Game Design, enquanto a Seção 3 apresenta o Pensamento Computacional. Já a Seção 4 descreve a metodologia utilizada, enquanto os resultados são apresentados na Seção 5. Por fim, as considerações finais são tratadas na Seção 6.

\section{Game Design}

Conforme o autor de [Pedersen, 2013], o documento de game design do jogo, ou GDD (game design document) é uma ferramenta textual que descreve todas as características de um jogo, desde informações básicas, conceitos, passando por personagens e cenários, até informações mais detalhadas sobre o jogo.

Perucia et al. (2005) afirmam que o GDD pode ser definido como "um documento que descreve as características do game design de modo abrangente". Já o autor de [Schuytema, 2008, p. 100] considera o game design como o coração e a alma de todos os documentos que giram em torno de um game em desenvolvimento e ressalta a importância dele, por apresentar uma descrição detalhada do jogo. Vale ressaltar que o game design é uma das primeiras etapas no desenvolvimento de jogos e que este documento pode ser modificado em qualquer momento da produção dos jogos.

\section{Pensamento Computacional}

O conceito de Pensamento Computacional foi inicialmente relatado por [Wing, 2006] para tratar da Ciência da Computação e de suas aplicações, que está relacionado desde a estruturação do raciocínio lógico até o comportamento humano e na resolução de problemas. Este tipo de pensamento pode ser visto nos processos de leitura, escrita e matemática como parte integrante da habilidade analítica das crianças desde a idade infantil. Outros autores como [Gomes e Melo, 2013] afirmam que o Pensamento Computacional tem uma contribuição na resolução de problemas, possibilitando que os indivíduos possam utilizar a computação nas diversas ações do cotidiano. 
Assim, os conceitos do Pensamento Computacional estão nos princípios da computação e não em suas tecnologias. Peter Lee em National Research Council (2010) descreveu o Pensamento Computacional como o estudo de mecanismos da inteligência humana que podem descrever aplicações e modelos que ajudem a tratar a complexidade.

O Pensamento computacional também pode ser definido como o pensamento analítico, compartilhando: com a matemática, a resolução de problemas; com a engenharia, modelagem e projeto; e com a ciência, a compreensão sobre computabilidade, inteligência, mente e comportamento humano [Wing 2008].

A Computer Science Teachers Association [CSTA 2011] ressalta um conjunto de competências que são trabalhadas nas atividades do pensamento computacional. Essas competências incluem a confiança em lidar com a complexidade; persistência ao trabalhar com problemas difíceis; tolerância em lidar com ambiguidade; capacidade de lidar com problemas em aberto; capacidade de se comunicar e trabalhar em grupo para atingir um objetivo.

\subsection{Testes de habilidades - Bebras Challenge}

O "Bebras" é uma iniciativa internacional com o objetivo de promover a Informática e o pensamento computacional entre estudantes de todas as idades. Os participantes geralmente são supervisionados por professores que podem integrar o desafio da Bebras em suas atividades de ensino. O desafio é realizado em escolas usando computadores ou dispositivo móvel [Bebras 2017].

A ideia do "Bebras" surgiu na Lituânia, pela Prof. Valentina Dagiene da Universidade de Vilnius. Bebras é uma palavra lituana para "castor". Um dos objetivos de Valentina Dagiene foi estabelecer a Bebras como uma iniciativa internacional em informática nas escolas. Desde do início, vários países europeus se juntaram ao "Bebras". De acordo com os fundadores, este teste pretende interessar crianças, jovens e adolescentes em problemas típicos de Ciência da Computação, não requerendo qualquer conhecimento como pré-requisito [Dagiene 2008].

Atualmente o desafio é organizado em mais de 30 países e tem o objetivo de levar estudantes de todo o mundo a serem entusiasmados com a computação. Cada participante do desafio recebe 45 minutos para responder 15 questões de múltipla escolha que se concentram no tema de pensamento computacional e lógico.

\section{Metodologia}

Para a execução deste trabalho, realizou-se inicialmente uma pesquisa de caráter exploratório de instrumentos e testes avaliativos que trabalhassem o pensamento computacional. O desafio Bebras foi o selecionado por ser um teste renomado e que já é adotado por mais de 30 países no Mundo.

Realizou-se então um estudo exploratório na produção de game designs que trabalhassem pensamento computacional. Documentos de game design foram produzidos e analisados, junto com formulários e observações realizadas. Na seção 
seguinte, é apresentado o planejamento do estudo exploratório como também as fases realizadas.

\subsection{Fases do estudo}

O estudo exploratório foi organizado nas seguintes fases:

1. Chamada para os alunos para a aplicação da pesquisa;

2. Selecionar questões do Teste Bebras;

3. Explicação e apresentação do Documento de Game Design;

4. Criar o documento de Game Design Educativo;

As fases são descritas a seguir. A fase 1 e 2 ocorreu em paralelo ao longo de duas semanas, enquanto as fases 3 e 4 ocorreu de forma sequencial na manhã de um único dia, ou seja, em 4 horas contínuas.

Fase 1: Chamada para os alunos para a aplicação da pesquisa: Nesta etapa os pesquisadores do laboratório divulgaram no portal da universidade uma chamada para alunos dos cursos de graduação que tivessem interesse em criar o game design para Jogos Digitais de Pensamento Computacional. Como resultados inscreveram-se e compareceram 19 alunos de graduação de diferentes áreas, conforme descrito na próxima seção.

Fase 2: Selecionar questões do Teste Bebras: Nesta etapa, dois pesquisadores selecionaram três questões do desafio Bebras referentes ao ano de 2016, com graus de dificuldades diferenciados: fácil, médio e difícil, classificação dada pelo próprio Bebras. As questões selecionadas foram: Bike Paths, Broken Window e Blossom. Para cada questão existem respostas e comentários disponibilizados pelo caderno de resposta do teste Bebras, os quais foram utilizados no estudo para facilitar o entendimento da questão e consequentemente a criação do game design. Na Tabela 1 são apresentadas as questões selecionadas com seus respectivos enunciados.

Tabela 1: Questões selecionadas do desafio Bebras.

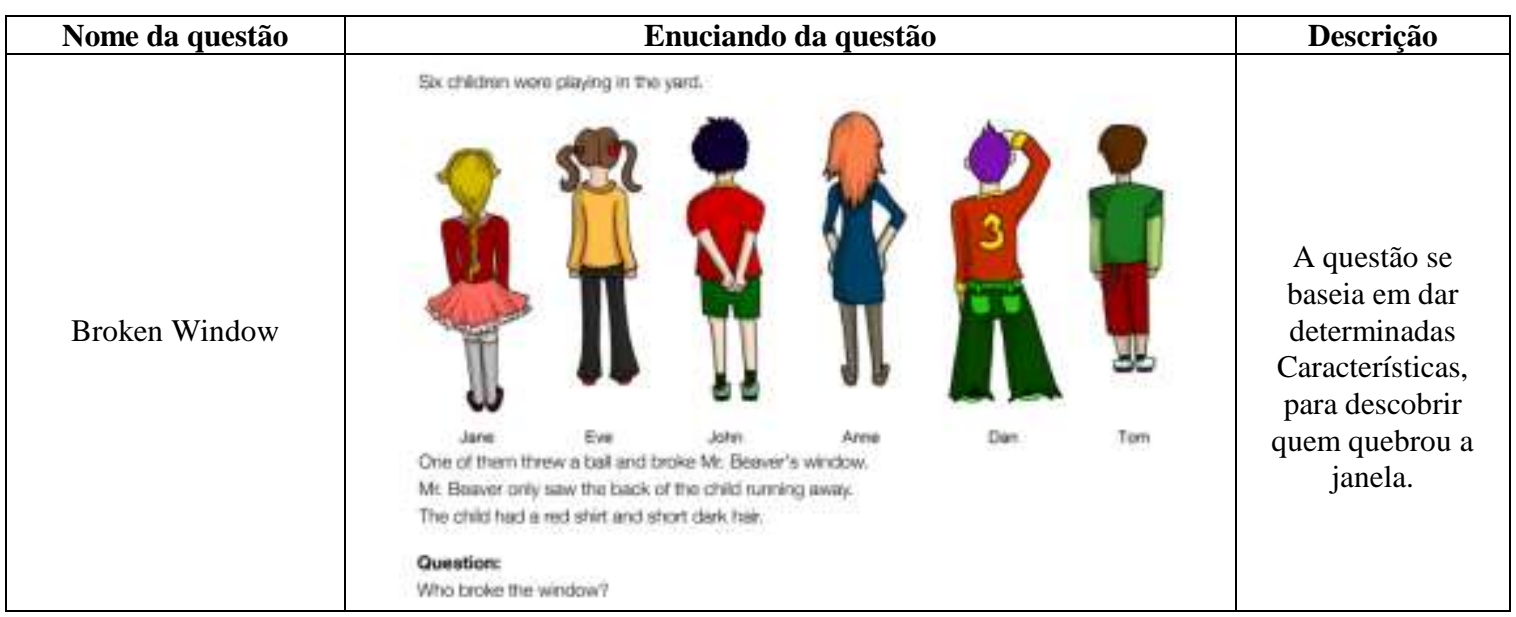




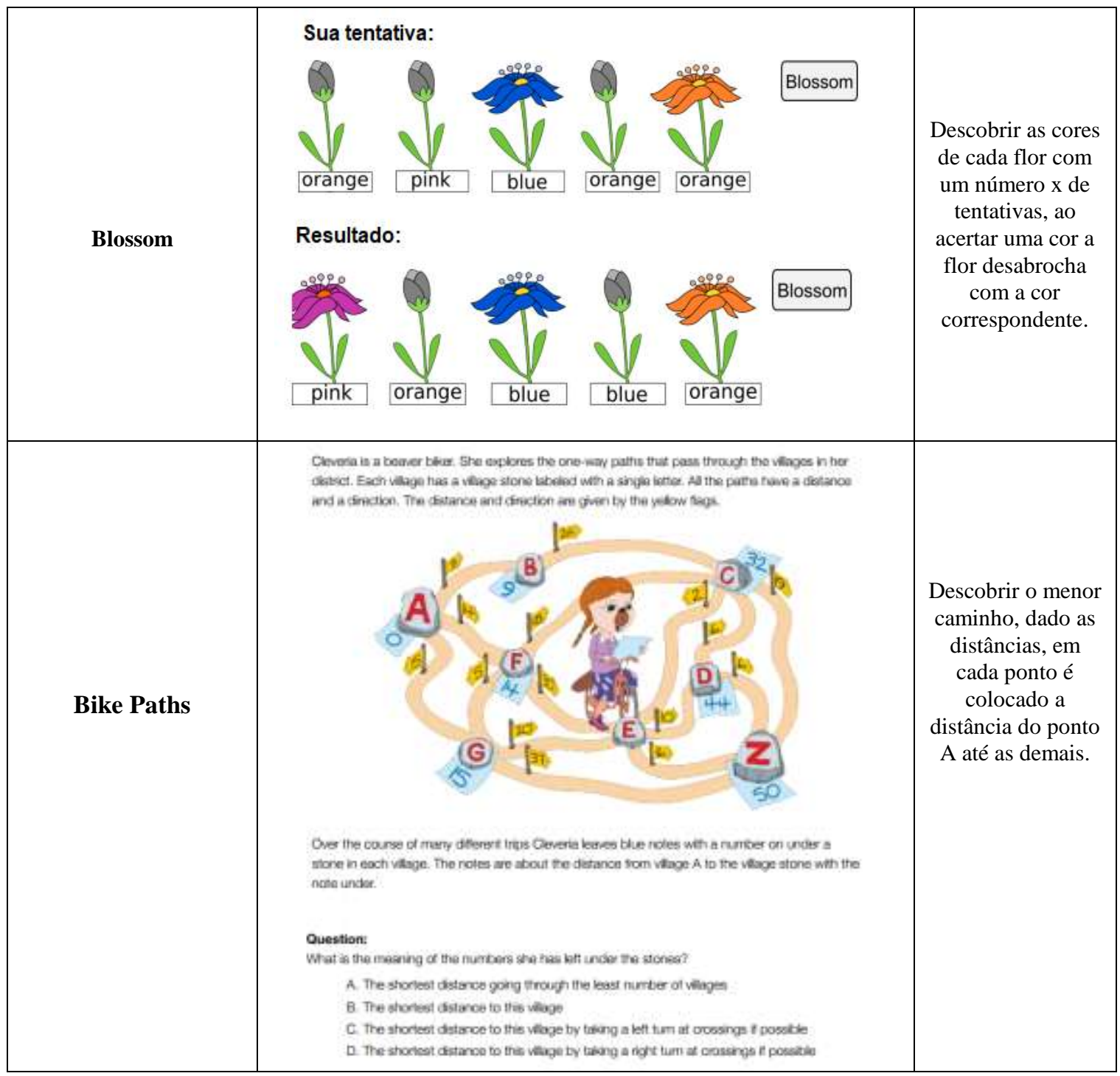

Fase 3: Explicação e apresentação do Documento de Game Design: Esta etapa foi aplicada no início do dia marcado com os alunos inscritos para o desenvolvimento do estudo. Por ter alunos de diferentes graduações, foi explicado como fazer um documento de Game Design apresentando um modelo de exemplo utilizado pelos os pesquisadores, facilitando assim a compreensão dos alunos.

Fase 4: Criar o documento de Game Design Educativo: Nesta etapa cada aluno de forma individual elaborava suas ideias de jogos a partir das questões e produziam os documentos de Game design.

\subsection{Perfis dos alunos}

Um formulário foi passado no início do estudo para verificar o perfil e as experiências prévias dos estudantes. Os detalhes são apresentados a seguir. O estudo contou com a participação de 19 estudantes com previsão de término de curso entre 2017.2 e 2020. 
Dos 19 alunos participantes, 17 são homens e 2 são mulheres. O perfil dos alunos que se interessaram por participar da pesquisa era diferente, pois os inscritos eram graduandos de diferentes cursos. A seguir é apresentada a tabela 2 referentes à distribuição dos alunos entre seus cursos.

Tabela 2. Cursos respectivos dos alunos inscritos.

\begin{tabular}{|c|c|c|}
\hline \multirow{4}{*}{$\begin{array}{c}\text { Perfil } \\
\text { dos }\end{array}$} & Curso & Quantidade alunos \\
\cline { 2 - 3 } Alunos & Tecnologia da Informação & 14 \\
\cline { 2 - 3 } & Química & 1 \\
\cline { 2 - 3 } & Licenciatura em Filosofia & 1 \\
\cline { 2 - 3 } & História & 1 \\
\cline { 2 - 3 } & Licenciatura em Física & $\mathbf{1 9}$ \\
\cline { 2 - 3 } & Total de Alunos & \multicolumn{2}{|c|}{} \\
\hline
\end{tabular}

Sobre experiência prévia, os estudantes poderiam relatar se possuíam algum contato ou experiência com jogos digitais, seja em disciplinas ou até mesmo na produção de jogos comerciais. Dos 19 alunos inscritos, apenas 3 relataram não possuir nenhuma experiência, ou seja, nunca tiveram contanto com universo dos jogos digitas. O gráfíco abaixo mostra esse percentual.

\section{Gráfico 1: Experiência com Jogos.}

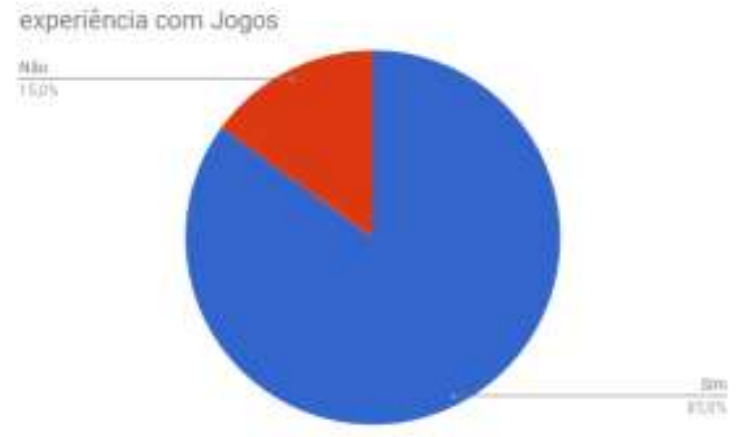

\section{Análise dos resultados}

Nesta seção são apresentados os resultados referentes à aplicação do estudo no laboratório com os alunos. Os resultados são provenientes das analises realizadas por meio de observações do comportamento dos alunos, da produção dos documentos de game design e nos formulários aplicados.

\section{Qual a qualidade dos game design produzidos?}

Os documentos de game design produzidos foram avaliados por 5 pesquisadores do laboratório com experiência na criação de game design. Foram verificados os seguintes aspectos de avaliação: baseados nas questões do Teste Bebras; criatividade na criação do enredo do jogo; preenchimento adequado do Game Design (GDD). 
Tabela 3.Critérios de avaliação do Game design

\begin{tabular}{|c|c|}
\hline Número do Critério & Critérios de avaliação do game design \\
\hline 1 & Baseados nas questões do Teste Bebras \\
\hline 2 & Criatividade na criação do enredo do jogo \\
\hline 3 & Preenchimento adequado do Game Design \\
\hline
\end{tabular}

No critério 1, verificamos se os game designs realmente adotaram como base o Teste Bebras. Nesse ponto, 14 alunos conseguiram realizar a criação do game design inspirados nas questões do teste. Entretanto, quatro alunos não compreenderam as explicações e acabaram levando em consideração outros instrumentos na criação do seu game design. Alguns alunos ao invés de criar os Games Designs das questões a partir do Teste Bebras procuraram outros instrumentos para realizar o game design, como por exemplo: questão do Enem, outros testes de avaliações e jogos já conhecidos no mercado.

Com relação ao critério 2, de criatividade, percebesse que relacionando o contexto de seus conhecimentos específicos em disciplinas como física, matemática, meio ambiente, contribui no processo criativo de desenvolver ideias para a criação do jogo. Com isso, acabou por agregar valor criativo e pedagógico aos game design das questões.

No critério 3, preenchimento do Documento de Game design, todos os alunos realizaram o preenchimento adequado do documento. Neste campo, foi analisado também a organização, explicação e a qualidade no preenchimento. Abaixo, segue exemplo de um o campo descrição, referente a o documento do game design realizado por um aluno.

Tabela 4. Exemplo do campo "descrição" do Game Design da questão Broken Window.

\begin{tabular}{|l|l|}
\hline DESCRIÇÃO: & $\begin{array}{l}\text { O jogador será um membro da Polícia Espacial. Uma jovem alien será assaltada e } \\
\text { dirá as características do assaltante. Essa informação aparecerá no canto direito } \\
\text { inferior da tela. Aparecerão, então, vários E.T.s num cenário, que são os "suspeitos". } \\
\text { Clicando num suspeito, o jogador irá abordá-lo. Se ele for o criminoso, será preso; } \\
\text { mas se for um civil inocente, o jogador perderá 1 chance de abordagem. Se ele } \\
\text { abordar } 3 \text { inocentes, será demitido (Game Over). Para achar o criminoso, o jogador } \\
\text { deverá encontrar o suspeito que tem características que batem com a descrição dada } \\
\text { pela vítima. }\end{array}$ \\
\hline
\end{tabular}

\section{Qual o esforço necessário para realizar a produção dos game design?}

Para avaliar o esforço dos alunos na criação do documento de game design foi dado inicialmente aos alunos o tempo de 4 horas para realizar o game design das três questões passadas. Porém, dentre os 19 alunos, apenas 31,6\% conseguiram terminaram no tempo estipulado. Os demais $68,4 \%$ dos alunos solicitaram mais tempo para terminar os game designs, alegando pouco tempo para a atividade. O gráfico a baixo ilustra o horário e a porcentagem referente à quantidade de alunos.

\section{Gráfico 2: Horários da Saída dos alunos.}




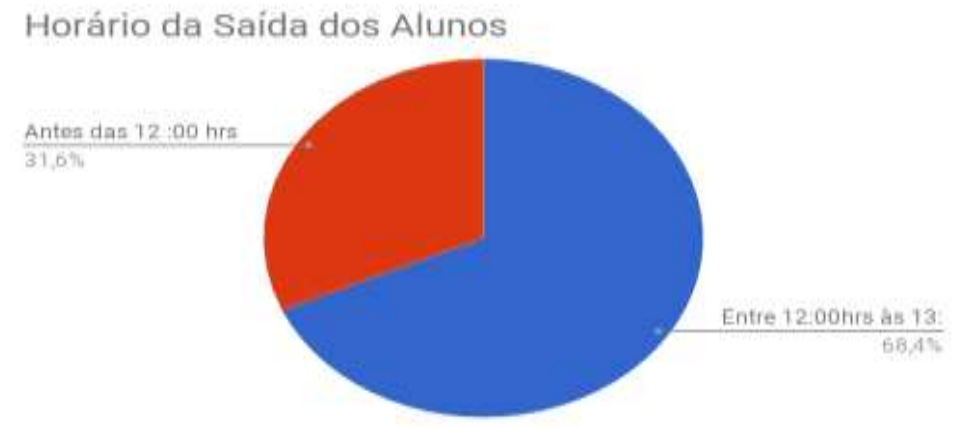

A partir desta questão, pode-se perceber que o tempo determinado para o estudo foi insuficiente, pois a maioria dos alunos como mostra o gráfico necessitaram de mais tempo. O estudo inicialmente foi estipulado de 4 horas, iniciando às 8 horas e terminando às 12 horas, porém durante a aplicação dos estudos alguns alunos solicitaram mais tempo e os pesquisadores dispuseram de mais 1 hora e com isso, dando assim um tempo de 5 horas. Além disso, mais tempo deve implicar em uma melhoria na qualidade do game design ou de viabilizar melhor o processo de criatividade, sem a pressão do tempo.

Nesta questão analisamos também o relato dos alunos, que ressaltaram como as questões selecionadas do Teste Bebras contribuíram para que não houvesse tanto esforço para delimitar o escopo a qual o game design seria criado. Desta forma, as questões ajudaram no processo de criação do game design e até mesmo no processo de criatividade. Porém, em contrapartida, alguns alunos não conseguiram concluir os 3 (GDD) questões, mesmo com a hora a mais adicionada.

\section{Quais as dificuldades apresentadas pelos alunos?}

Por meio das observações na aplicação do estudo como também, pelo formulário respondido e pelos game designs produzido, foi visto que alguns alunos apresentaram dificuldade como:

- Compreender o objetivo do estudo, que era de utilizar as questões do Teste Bebras como base para realizar os games design. Dos 19 alunos 4 acabaram por pesquisar em outros instrumentos avaliativos questões para produzir o Game Design. Vale ressaltar que estes quatros apresentavam a semelhança de não serem do curso de Tecnologia da Informação, podendo assim, ser uma relação que pudessem tê-los prejudicado na compreensão.

- Durante estudo alguns alunos apresentaram dúvidas e solicitaram o esclarecimento dos pesquisadores mediadores. Cinco alunos fizeram em vários momentos a mesma pergunta sobre como usar as questões para elaborar o game design.

- Alguns alunos solicitaram mais tempo para realizar os games design, alegando que tempo estava insuficiente.

- Apresentaram dificuldade com alguns campos do game design, acabando por deixá-los sem preenchimento.

- Dentre as questões apresentadas, os alunos relataram maior dificuldade de compreensão para criar o game design com a questão 3 - Bike Paths. 
- Dos 19 alunos, cinco alunos deixaram de fazer o Game Design de um dos três jogos que deveria ser feito, como apresentado no gráfico 2.

\section{Gráfico 3: Quantidade alunos que realizaram a criação dos Games Designs.}

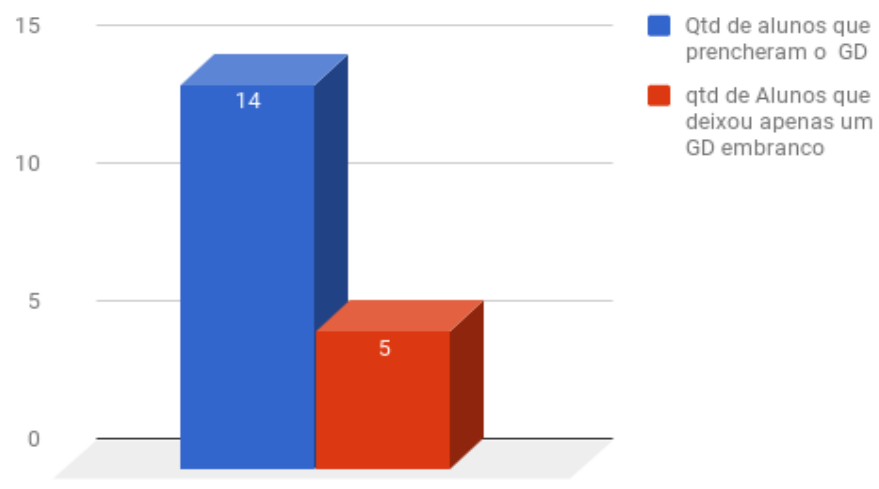

\section{Conclusões}

Este trabalho apresentou um estudo exploratório de como produzir a criação de game design de Jogos Digitais de Pensamento Computacional a partir de questões do Teste internacional Bebras. Podemos perceber que alunos de diferentes graduações se interessaram por participarem do convite do estudo e como a utilização de uma base para gerar o game design pode ajudar aos alunos a criarem o game design.

Com a utilização de questões do Teste Bebras, pode-se garantir a criação dos game design voltados ao pensamento computacional. Essa fonte de inspiração ajudou os alunos a produzir de maneira mais concisa e eficaz os documentos, pois muitos alegaram a contribuição das questões selecionadas, que os ajudaram no processo. De fato, os participantes relataram que se não houvesse às questões levariam mais tempo e consequentemente mais esforço para estabelecerem um escopo para criação dos seus games designs.

A contribuição deste trabalho se dá no contexto atual de cada vez mais trabalhar os aspectos educacionais e estimular o pensamento computacional na produção dos jogos, tendo em vista sua importância no ambiente educacional de estimular a resolução de problemas e desenvolver raciocínio lógico. O trabalho também investiga a produção de game design a partir de questões de testes avaliativos reconhecidos, como uma forma de facilitar esse processo.

Esperamos que este estudo possa de alguma forma servir de auxílio para os atuais e futuros envolvidos com o tema, bem como, contribuir para o avanço de buscar métodos para produção de game design na produção de jogos digitais de pensamento computacional. Pretendemos assim, que com este trabalhos incentive pesquisas para difundir o pensamento computacional como possibilitar à aproximação o universo dos jogos educativos, consequentemente de meios para aplicações da tecnologia aos alunos de outras áreas.

Como trabalhos futuros, a proposta é elaborar um estudo mais completo selecionando mais questões do teste Bebras como também de outros testes avaliativos. 
Pretende-se também aumentar o número de alunos para realização de pesquisa como estender o estudo em outras instituições, bem como fomentar não só a criação de game designs, mas também do desenvolvimento e aplicação desses jogos na educação.

\section{Referências}

Balasubramanian, Nathan; Wilson, Brent G. Games and Simulations. In: Society For Information Technology And Teacher Education International Conference, 2006. Proceedings...v.1. 2006.

CSTA\&ISTE (2011). Computational thinking teacher resources. http://csta.acm.org/curriculum/sub/compthinking.html. acesso: outubro/2017

Gomes, T. e Melo, J. (2013). O pensamento computacional no ensino medio: Uma abordagem blended-learning. In Anais do 21o WEI -XXXIII CSBC. Maceio, ALBrasil.

Kenski, Vani Moreira. (2013) Tecnologias e tempo docente. São Paulo: Papirus Editora

Prensky, M., 2001. Digital game-based learning. New York, McGraw-Hill.

R. Pedersen. Game design foundations. 1.ED. Sudbury: Wordware publishing, INC. 2003.

A.S. Perucia, A. C. Berthêm, G. L. Bertschinger e R. R. C. Menezes. Desenvolvimento de Jogos Eletrônicos: teoria e prática. São Paulo: Novatec Editora, 2005.

V. Dagiene and G. Futschek, "Bebras International Contest on Informatics and Computer Literacy: Criteria for Good Tasks," in Informatics Education - Supporting Computational Thinking, ISSEP 2008, Torun, Poland, July 1-4, 2008: Springer, 2008, pp. 19-30

Wing, J. M. (2006). Computational thinking. Communications of the ACM, 49(3):3335. 